\title{
Semantic Interoperability in Biomedicine and Health Care II.
}

\author{
Štēpán Svačina, Jana Zvárová
}

The first issue of the European Journal for Biomedical Informatics in 2011 publishes peer-reviewed papers of students of the doctoral study at the 1st Faculty of Charles University in Prague. These papers were also presented as lectures given by Ph.D. students during the second workshop on the topic "Semantic interoperability in biomedicine and health care" held on November 24th, 2011 in Prague. The first workshop on the same topic was held on November 18th, 2010 in Prague and all papers of the first workshop were published in English and Czech languages in the European Journal for Biomedical Informatics Vol.6, Issue 1, 2010.

Semantic interoperability and the ability to obtain specific information by usage of technical means are essential conditions for the utilization of other telemedicine technologies and eHealth. The ability of systems to understand exchanged data (semantic interoperability) requires using the same terminology (i.e. classification systems and nomenclatures) and using the same language for communication and its recording (data standards). If information in biomedicine and health care is shared using a free text, a prerequisite for semantic interoperability is the access to its meaning. Existing standards (e.g. EN 13606) suppose the use of globally unique and uniquely defined terms that can be without much difficulty transferred to other classifications (e.g. by means of the Unified Medical Language System). Probably the best applicable general classification system for healthcare is SNOMED CT. It has arisen by a combination of American SNOMED (created by the Association of American Pathologists) and British Clinical Terms ("Read Codes"). In connection with this merger the International Health Terminology Standards Development Organization (IHTSDO), with the residence in Denmark, has been founded in 2007. IHTSDO is a not-for-profit association that develops and promotes use of SNOMED CT to support safe and effective health information exchange. SNOMED CT is a clinical terminology and is considered to be the most comprehensive, multilingual healthcare terminology in the world. SNOMED CT is now being used in a number of information systems for recording of clinical information within patient records. It is expected from modern information systems to work effectively with information and to exchange it mutually.

The task of the workshop supported by the project of the specific research at the 1st Faculty of Medicine of Charles University was also to present selected terms from papers of students and to make their description in English and classification by the SNOMED CT. Then the translations of these findings to the Czech language were presented at the workshop. 